\title{
Engineering Anti-bunching via Photon Blockade in Photonic Crystal Cavity-Quantum Dot Systems
}

\author{
Arka Majumdar, Andrei Faraon, Jelena Vuckovic \\ E. L. Ginzton Laboratory, Stanford University, Stanford, CA-9430 \\ e-mail: arkam@stanford.edu
}

\begin{abstract}
Methods to improve single photon generation via photon-blockade in a photonic-crystal cavity with a strongly coupled quantum-dot are presented. With realistic system parameters, significant improvement in second-order-auto-correlation $\mathrm{g}^{2}(0)$ (from 0.93 to 0.79 ) is achieved. (C)2009 Optical Society of America

OCIS codes:_(050.5298) Photonic crystals; (270.5585) Quantum information and processing
\end{abstract}

Growing interest in making scalable Quantum Information Processors (QIP) combined with modern semiconductor fabrication technology resulted in numerous proposals for making cavity QED system in semiconductors. One of the major requirements of QIP is a non-classical light-source. We have previously demonstrated photon blockade in a photonic crystal - quantum dot (PhC-QD) system [1]; such an effect could be employed to make non-classical light sources on chip, but due to sub-optimal system parameters, only a limited blockade effect was achieved. Here we analyze a possibility of achieving a stronger photon blockade, and thus of achieving a stronger antibunching upon transmitting a coherent light beam tuned to the first order dressed states through such a system, within the constraints of the state of the art technology.

If a QD is placed inside a cavity with two degenerate and orthogonal modes (which can be theoretically obtained in a cavity with C6 symmetry) and one of the modes is coherently driven by a laser, then the corresponding Hamiltonian (in rotating wave approximation with driving laser frequency) is given by:

$$
H=\Delta_{c} a^{\dagger} a+\left(\Delta_{c}+\delta\right) b^{\dagger} b+\Delta_{a} \sigma_{z}+i g_{o}\left(\sigma_{-} a+\sigma_{+} a^{\dagger}+\sigma_{-} b+\sigma_{+} b^{\dagger}\right)+\Omega\left(a+a^{\dagger}\right)
$$

Here, $a$ and $b$ are the annihilation operators for the two cavity modes; $\Delta_{\mathrm{c}}$ is the detuning between laser frequency and mode $a, \delta$ is the detuning between mode $a$ and $b$ (for a degenerate cavity $\delta=0$ ), $\Delta_{\mathrm{a}}$ is detuning between driving laser and the QD resonance frequency; $\sigma_{-}, \sigma_{+}$are the lowering and raising operators for the QD; $2 \sigma_{z}=\left[\sigma_{-}, \sigma_{+}\right] ; \Omega$ is the Rabi frequency of the driving laser; $g_{o}$ is the coupling strength between cavity modes and QD (same strength for both modes are assumed). We assume that in the experiment, light from only mode $a$ (or mode $b$ ) can be collected as the two modes can be distinguished by polarization. By solving Master Equation with this Hamiltonian (1), and using damping parameters (cavity decay $\kappa$ and dipole decay $\gamma$ ) from Ref. [1] (and assuming no detuning is present between cavity modes and the QD), $\mathrm{g}^{2}(0)$ for mode $a$ is obtained, (Figure 1A, blue curve). It should be noted that as opposed to single cavity mode situation studied in Ref. [1] (Fig. 1A red curve), three antibunching points are obtained. If the cavity modes and QD are all resonant (frequency $\omega_{\mathrm{c}}$ ), then the three energy levels correspond to $(m+n+1 / 2) \omega_{c}$ and $(m+n+1 / 2) \omega_{c} \pm \sqrt{4 g_{0}{ }^{2}(m+n+2)}$, where $\mathrm{m}$ is the initial number of quanta in the mode $a, \mathrm{n}$ is the quanta in the mode $b$, and 1-quanta in the QD. Antibunching points $\mathrm{B}$ and $\mathrm{C}$ are due to ordinary Jaynes-Cummings ladder (formed by the last two energy levels) as explained by [2]. Antibunching point A (which is more antibunched relative to single mode case, with $\mathrm{g}^{2}(0)=0.79$ compared to $\mathrm{g}^{2}(0)=0.93$ ) is obtained when the laser frequency is tuned to cold-cavity frequency $\omega_{c}$. This happens as the successive levels at energy $(m+n+1 / 2) \omega_{c}$ are of opposite parity and hence transition between them is not allowed. For practical implementation, it is difficult to obtain simultaneous degeneracy and orthogonality in high quality $\mathrm{PhC}$ cavity modes and hence simulations are also done with non-degenerate and non-orthogonal cavity modes. To simulate nonorthogonality of modes, a coupling term between two cavity modes is added in the Hamiltonian in Eq. 1 with coupling strength $\mathrm{g}_{\mathrm{ab}}$ (Coupling term: $g_{a b}\left(a^{+} b+a b^{+}\right)$). Figure 1B shows the $\mathrm{g}^{2}(0)$ value at anti-bunching point A with different mode detuning $\delta$ and coupling $g_{a b}$ values. The reduction of $g^{2}(0)$ with non-degeneracy of cavity mode is expected. For non-orthogonal modes, it is found that there is an optimum point. The mode $b$ gets photon only through QD when modes $a$ and $b$ are orthogonal. Hence the mode $b$ has more non-classical nature than mode $a$. When there is small leakage from $a$ to $b$ then the mode $a$ becomes more anti-bunched due to light from mode $b$. But increasing leakage ultimately makes both the modes more classical. 


\section{IThB7.pdf}

Another way to improve the antibunching, is to cascade two single mode PhC-QD systems but at the cost of reducing the efficiency of the source. As a non-classical light cannot be expressed in a finite Fock-state basis, hence the cascaded system is modeled as a single system (with coupling strength $\eta$ ) [3], and that the coupling is such that light travels in one direction (i.e., only forward traveling wave is present). Simulation results are shown in Figure 2. It should be noted that, the $\mathrm{g}^{2}(0)$ is improved after cascading. Increasing $\eta$ does not help in reduction of $\mathrm{g}^{2}(0)$, but it increases the output intensity.

In conclusion, we analyzed two proposals for improving the anti-bunching of light by photon-blockade in a QD$\mathrm{PhC}$ cavity system as reported in [1]. If this elementary system can be improved (e.g., by pulse shaping of coherent light-source to increase coupling of photons to the cavity, or by increasing cavity Q-factor), then these two approaches will give even better performances than simulated here.

Financial support was provided by ONR Young Investigator Award, DARPA Young Faculty Award, SGF (T.I. Fellowship), Presidential Early Career Award for Engineers and Scientists (PECASE) and Army Research Office.

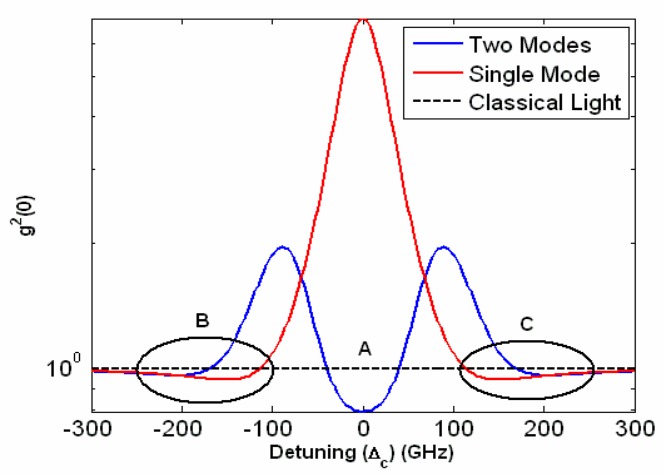

(A)

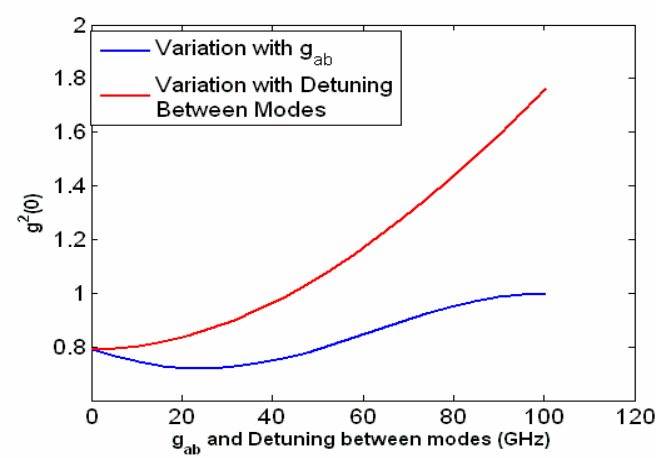

(B)

Figure 1: (a) $\mathrm{g}^{2}(0)$ for three different situations. In all these cases $\mathrm{g}_{0}=\kappa=16 \mathrm{GHz}, \gamma=\kappa / 80, \Omega=50$. Here it is assumed that cavity modes and the QD are resonant. The two modes are orthogonal and degenerate. (b) Variation of $\mathrm{g}^{2}(0)$ at point A with detuning between cavity modes and $\mathrm{g}_{\mathrm{ab}}$. Same damping parameters and driving term are used.

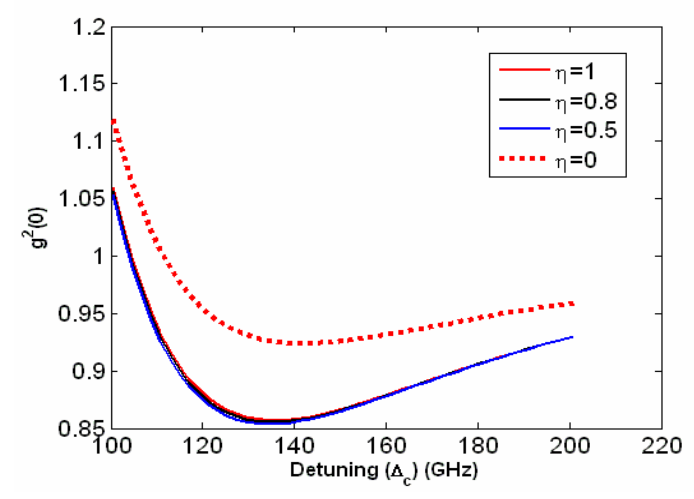

Figure 2: $\mathrm{g}^{2}(0)$ with $\Delta_{\mathrm{c}}$ for different coupling strength $\eta$. In both the cavities $\mathrm{g}_{0}=\kappa=16 \mathrm{GHz}, \gamma=\kappa / 80, \Omega=10$.

\section{References:}

[1] A. Faraon et. al.; Coherent generation of nonclassical light on a chip via photon-induced tunneling and blockade; Nature Physics; (2008).

[2] K. M. Birnbaum et. al.; Photon Blockade in an optical cavity with one trapped atom; Nature; vol. 436, 2005.

[3] C.W. Gardiner, P. Zoller; Quantum Noise; Third Edition; Springer. 